\title{
Impact of anti-HER2 therapy on overall survival in HER2- overexpressing breast cancer patients with brain metastases
}

\author{
R Bartsch 1,2, A Berghoff',2, U Pluschnig ${ }^{1,2}$, Z Bago-Horvath ${ }^{2,3}$, P Dubsky,4, A Rottenfusser 2,5, C DeVries ${ }^{1,2}$,

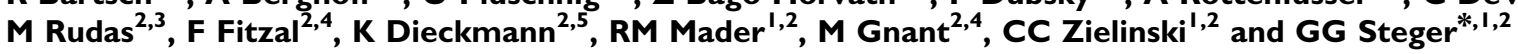 \\ 'Clinical Division of Oncology, Department of Medicine I, Medical University of Vienna, Waehringer Guertel I 8-20, Vienna I090, Austria; ${ }^{2}$ Comprehensive \\ Cancer Centre, Medical University of Vienna, Waehringer Guertel 18-20, Vienna 1090, Austria; ${ }^{3}$ Department of Pathology, Medical University of Vienna, \\ Waehringer Guertel 18-20, Vienna 1090, Austria; ${ }^{4}$ Department of Surgery, Medical University of Vienna, Waehringer Guertel 18-20, Vienna 1090, \\ Austria; ${ }^{5}$ Department of Radiotherapy, Medical University of Vienna, Waehringer Guertel 18-20, Vienna 1090, Austria
}

BACKGROUND: Trastuzumab-based therapy after diagnosis of brain metastases (BM) may improve survival due to prolonged systemic disease control. We investigated whether lapatinib may yield additional survival benefit.

METHODS: Eighty patients with BM from HER2-positive breast cancer were identified. Karnofsky Performance Score (KPS) of at least 70 was required. We included a control group of 37 patients treated before 2003, when continuation of trastuzumab after diagnosis of BM was not yet recommended. Remainders received either trastuzumab or lapatinib and trastuzumab (either concomitantly or sequentially) with or without chemotherapy.

RESULTS: Median overall survival (OS) in patients receiving trastuzumab after diagnosis of BM was I 3 months; corresponding numbers were 9 months in patients treated with chemotherapy, and 3 months with radiotherapy alone. Median OS was not reached in the lapatinib group. Addition of lapatinib prolonged $O S$ over trastuzumab alone $(P=0.002)$. After correction for potential confounders, lapatinib therapy remained an independent positive predictor for survival $(H R \quad 0.279 ; P=0.012)$.

INTERPRETATION: This retrospective single-centre study suggests that the introduction of lapatinib improved survival in patients with BM from HER2-positive breast cancer. Patients with KPS $\geqslant 70$ may benefit when treated with lapatinib in addition to trastuzumab after completion of local therapy.

British Journal of Cancer (2012) 106, 25-31. doi:10.1038/bjc.201 I.531 www.bjcancer.com

Published online 29 November 2011

(c) 2012 Cancer Research UK

Keywords: brain metastases; breast cancer; HER2-positive disease; lapatinib; trastuzumab

Among solid cancers, breast cancer is the second most common cause of central nervous system (CNS) metastases and the most common cause of carcinomatous meningitis (Weil et al, 2005; Stemmler and Heinemann, 2008). Various risk factors were identified: young age, advanced and hormone receptor-negative disease, short disease-free interval, high disease burden, and visceral metastases (Miller et al, 2003; Evans et al, 2004; Lin et al, 2004; Slimane et al, 2004; Weil et al, 2005). A marked increase in the incidence of brain metastases (BM) was observed in HER2-positive patients over the last decade. This finding is usually attributed to the survival benefit associated with the introduction of trastuzumab, a monoclonal antibody targeting HER2; furthermore, trastuzumab, due to its molecular size, has limited ability to pass through the blood-brain barrier, rendering the CNS an important tumour cell sanctuary (Bendell et al, 2003; Clayton et al, 2004; Shmueli et al, 2004; Burstein et al, 2005; Viani et al, 2007). In favour of that notion, the rate of patients with brain as first site of disease progression is increasing by time (Yau et al, 2006).

Treatment for BM consists of corticosteroids, whole brain radiotherapy (WBRT) as well as neurosurgical resection, radiosurgery,

*Correspondence: Dr GG Steger, E-mail: guenther.steger@meduniwien.ac.at Received I 4 July 20 I ; revised 31 October 201 |; accepted 8 November 201 I; published online 29 November 201 I and boost irradiation as indicated (Borgelt et al, 1980; Bindal et al, 1993; Lohr et al, 2001). Whole brain radiotherapy yields symptomatic and clinical responses in $\sim 50 \%$ of patients, while survival remains dismal at 6 months (Lutterbach et al, 2002; Broadbent et al, 2004). Systemic therapy has limited impact on BM (Rosner et al, 1986). While three recent studies reported better survival outcomes when patients with $\mathrm{BM}$ received further trastuzumab after completion of local therapy, it is assumed that the impact on overall survival (OS) is due to control of systemic disease rather than brain lesions (Lower et al, 2003; Kirsch et al, 2005; Bartsch et al, 2007). Lapatinib, a small molecule tyrosinekinase inhibitor of EGFR and HER2, was recently approved for the treatment of HER2-positive metastatic breast cancer. Due to its small molecular size, lapatinib may pass the blood - brain barrier, opening possibilities for medical treatment and prophylaxis of CNS metastases (Cameron et al, 2008; Lin et al, 2008). Indeed, two phase II studies conducted in patients with established BM reported a modest yet significant activity of lapatinib by indicating a volumetric reduction in the size of brain lesions (Lin et al, 2008, 2009). Importantly, the 2-year OS was higher in patients with BM responding to lapatinib-based therapy as compared with those with stable or progressive CNS disease (66\% vs $44 \%$ ). This suggests that with improved systemic disease control, better local control of brain lesions yields additional survival benefit. 
Based upon those assumptions, we investigated whether lapatinib-based treatment may improve survival outcome in patients with BM from HER2-positive breast cancer. Accordingly, we compared patients receiving lapatinib and trastuzumab (either sequentially or concomitantly) after completion of local therapy with individuals who only received trastuzumab plus/minus chemotherapy and a historical control group of HER2-positive subjects without any further targeted therapy.

\section{PATIENTS AND METHODS}

Patient data were collected at the Comprehensive Cancer Centre, Medical University of Vienna. This retrospective analysis was approved by the local ethics committee.

\section{Patients}

Data from all consecutive patients who were treated with local therapy for BM from HER2-positive breast cancer from 2003 until 2010 who received trastuzumab and/or lapatinib after completion of local therapy for BM were retrieved from a breast cancer database (group A). Patients without further systemic therapy or Karnofsky Performance Score (KPS) $<70$ were not included to avoid an inclusion bias, as low KPS is a known negative predictor of OS. In a second step, data were retrieved from patients who received local treatment for BM between 1998 and 2002, and served as control; 2002 was chosen as cutoff, as from 2003 onwards continuation of trastuzumab treatment after diagnosis of BM was generally recommended. Again, patients with KPS $<70$ or incomplete data sets were excluded (group B). Within group B, patients either received chemotherapy after completion of local treatment or no further systemic therapy at all. This decision was taken at the discretion of the treating physician and patients without further chemotherapy were thought to have no meaningful systemic treatment option left. In total, 80 patients were available for this retrospective analysis (Figure 1).

\section{Treatment plan and patient evaluation}

In patients with $>3$ metastases, WBRT was applied at a 6-MV linear accelerator (LINAC) by lateral opposed fields. Total dose prescribed was 30 Gray (Gy) in 10 fractions of $3 \mathrm{~Gy}$. In case of one to three metastases $\leqslant 2 \mathrm{~cm}$, a stereotactic boost was applied at a Gamma knife (16-20 Gy on the $50 \%$ isodose), or at a 6-MV LINAC ( $20 \mathrm{~Gy}$ on the $80 \%$ isodose). In case of tumour size $>2 \mathrm{~cm}$, two times 10 Gy were applied at a 6-MV LINAC. Boost irradiation was applied either alone or in combination with WBRT. In selected cases, prior neurosurgical resection had been performed.

Trastuzumab was administered at a dose of $6 \mathrm{mg} \mathrm{kg}^{-1}$ body weight every 3 weeks after a loading dose of $8 \mathrm{mg} \mathrm{kg}^{-1}$ body weight on the first day of treatment. Lapatinib was administered at a daily fixed dose of $1000 \mathrm{mg}$ (in combination with trastuzumab), $1250 \mathrm{mg}$ (in combination with capecitabine), or $1500 \mathrm{mg}$ (as single agent) with appropriate dose reductions if necessary.

HER2 status was assessed using the HercepTest (Dako A/S, Glostrup, Denmark) or dual colour fluorescent in-situ hybridisation (FISH; PathVision HER2 DNA probe kit, Vysis Inc., Downers Grove, IL, USA). Tumours were classified as HER2 positive if they had a staining intensity of +++ on the HercepTest; if a score of ++ was gained, tumours were reanalysed by FISH. In patients with neurosurgical resection of BM, HER2 status was reassessed from the CNS lesion. From 2008 onwards, HER2 status was generally reassessed at the time of first diagnosis of metastatic disease. In those patients, a biopsy from one metastatic site was taken whenever feasible. In patients progressing before 2008, HER2 status was not reassessed and HER2 status of the primary tumour was used as surrogate.

Oestrogen and progesterone receptor status were assessed by immunohistochemistry (ER $\alpha$ antibody, clone 1D5; Dako A/S and PgR antibody; Dako A/S). Receptor expression was estimated as the percentage of positively stained tumour cells. Results were given as,,++++++ positive staining or negative staining, with a cutoff value of $<10 \%$ positive tumour cells.

All patients had symptomatic BM and no routine screening for CNS involvement was conducted. For baseline staging evaluations, all patients had CT scans of the chest and abdomen, mammography, and gynaecologic examination. Before initiation of trastuzumab or lapatinib treatment, echocardiography was mandatory, and patients with left ventricular ejection fraction (LVEF) $<50 \%$ were excluded. Restaging was performed every 3 months. Cranial MRI scans were performed 1 and 3 months after completion of local therapy, and every 3 months thereafter. In patients treated before 2003, cranial CT scans instead of MRI scans were repeatedly performed during follow-up, thus complicating the assessment of response and progression-free survival (PFS)

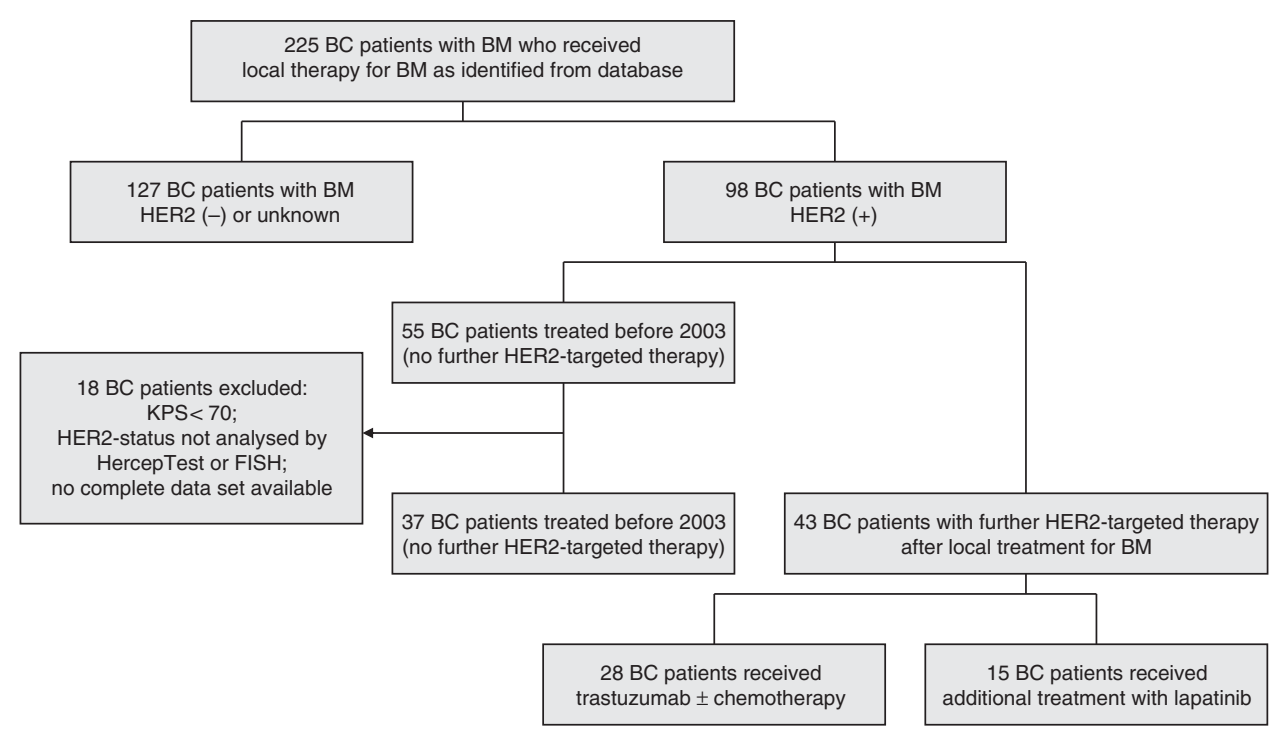

Figure I Patient cohort. $\mathrm{BC}=$ breast cancer; $\mathrm{BM}=$ brain metastases; HER2 $(+)=$ HER2 positive; HER2 $(-)=$ HER2 negative. 
within the CNS. Furthermore, restaging was not always conducted at regular 12-weekly intervals; therefore, no analysis of CNS response and PFS was performed.

\section{Statistical analysis}

Overall survival was chosen as primary study end point; OS was defined as interval from diagnosis of symptomatic BM until death of any cause and estimated using the Kaplan-Meier product limit method. To test for differences between respective OS curves, the log-rank test was used. $P$-values $<0.05$ were considered to indicate statistical significance.

The following variables were included in the univariate analysis of OS: type of systemic therapy after completion of local treatment (no further treatment, chemotherapy only, any treatment including trastuzumab, any treatment including lapatinib); pathological subtype (ductal $v s$ lobular); grading (grade 1 and $2 v s 3$ ); hormone receptor status; presence of visceral metastases; brain as only site of metastatic disease; presence of $>2$ metastatic sites outside the CNS; early diagnosis of BM $(<12$ months from diagnosis of breast cancer or metastatic disease); age $>65$ years at diagnosis of $\mathrm{BM}$; KPS (70 vs $>70)$; number of brain lesions $(1-3 v s>3)$.

A Cox proportional hazard model was used to correct for factors significantly $(P<0.05)$ or borderline significantly $(P<0.07)$ associated with OS on the univariate analysis.

Differences between demographic data are described with frequencies and percentages and were tested with the Fisher's exact test.

Data were analysed as of February 2011. All statistics were calculated using statistical package for the social sciences (SPSS) 17.0 software (SPSS Inc., Chicago, IL, USA).

\section{RESULTS}

\section{Patient characteristics}

Overall, 80 patients with KPS $>70$ treated for BM from HER2-positive breast cancer were identified from a breast cancer database. Fortythree patients received anti-HER2 treatment after completion of local therapy (group A). Within this cohort, 28 patients received trastuzumab plus/minus chemotherapy and another 15 were additionally treated with lapatinib. A total of 37 patients who were treated before 2003 and therefore received no further anti-HER2 treatment after completion of local therapy were available as control group for this analysis (group B). Within this group, 9 patients received chemotherapy while 28 had no further systemic treatment.

In the total sample of 80 patients, median age at diagnosis of BM was 53 years (range 28-77 years), and 14 patients were $>65$ years. Median KPS was 80 (range 70-100); 62 patients had KPS of $>70$. Median time to diagnosis of BM from diagnosis of breast cancer was 36 months (range $0-254$ months). In 9 patients, brain was the only site of metastatic disease, 56 had visceral metastases at the time BM were diagnosed, and 39 patients presented with more than two metastatic sites. Seventy-three patients received WBRT, with the remainders treated with boost irradiation only. An additional neurosurgical resection was conducted in 15 subjects. Median duration of trastuzumab therapy after completion of local therapy was 8.5 months, and median duration of lapatinib-based therapy was 8 months. Characteristics of the total patient sample are summarised in Table 1.

The only imbalance between both treatment groups (group A (further anti-HER2 therapy) and group B (no further anti-HER2 therapy)) was the percentage of patients treated with boost irradiation or radiosurgery alone without WBRT as local therapy for BM: 1 out of $37(2.7 \%)$ in the years before 2003 vs 6 out of $43(14 \%)$ thereafter (n.s.). This difference likely demonstrates a change in treatment routine and is unlikely to impact on survival. Patient characteristics separated for groups A and B are summarised in Table 2.
Table I Patient characteristics (total patient population)

\begin{tabular}{|c|c|}
\hline Characteristics & Patients \\
\hline Entered & $n=80$ \\
\hline Median age at diagnosis of BM (years) (range) & $53(28-77)$ \\
\hline Patients $\geqslant 65$ years & $n=14(17.5 \%)$ \\
\hline Karnofsky Performance Score (median) & 80 \\
\hline Patients KPS >70 & $n=62(77.5 \%)$ \\
\hline Hormone receptor positive & $38(47.5 \%)$ \\
\hline HER2 positive & $80(100 \%)$ \\
\hline Grading 3 & $58(72.5 \%)$ \\
\hline Invasive ductal carcinoma & $66(82.5 \%)$ \\
\hline \multicolumn{2}{|l|}{ Stage at primary diagnosis } \\
\hline Localised & $68(85 \%)$ \\
\hline Metastatic & $12(15 \%)$ \\
\hline Visceral disease & $56(70 \%)$ \\
\hline$>2$ Metastatic sites outside the CNS & $39(48.8 \%)$ \\
\hline Only BM & $9(11.3 \%)$ \\
\hline I -3 Brain metastases & $40(50 \%)$ \\
\hline Development of BM in $<12$ months & $23(28.8 \%)$ \\
\hline Adjuvant chemotherapy & $64(80 \%)$ \\
\hline Adjuvant endocrine therapy & $28(35 \%)$ \\
\hline Palliative chemotherapy & $60(75 \%)$ \\
\hline Palliative endocrine therapy & $19(23.8 \%)$ \\
\hline Trastuzumab before diagnosis of BM & $57(71.3 \%)$ \\
\hline Duration (months) (range) & $12.5(3-57)$ \\
\hline
\end{tabular}

Time to diagnosis of BM (from primary breast cancer diagnosis) Median (months) (range)

$36(0-254)$

Time to diagnosis of BM (from diagnosis of metastatic disease)

Median (months) (range)

Radiosurgery or boost irradiation without WBRT

Systemic treatment after local therapy for BM

Group A (further anti-HER2 therapy after diagnosis of BM)

$43(53.8 \%)$

$28(35 \%)$

Trastuzumab plus/minus chemotherapy

Lapatinib plus/minus trastuzumab plus/minus chemo

Group B (no anti-HER2 therapy after diagnosis of BM)

No further treatment

Chemotherapy only

$15(18.8 \%)$

$37(46.3 \%)$

$28(35 \%)$

$9(11.3 \%)$

Duration of trastuzumab treatment after local therapy for BM

Months (median) (range)

$8.5(2-37)$

Duration of lapatinib treatment after local therapy for BM Months (median) (range)

$8(2-23)$

Abbreviations: $\mathrm{BM}=$ brain metastases; $\mathrm{CNS}=$ central nervous system; $\mathrm{WBRT}=$ whole brain radiotherapy; KPS = Karnofsky Performance Score.

Table 2 Patient characteristics (separated for groups A and B)

\begin{tabular}{|c|c|c|c|}
\hline Characteristics & Group A & Group B & $P^{a}$ \\
\hline Entered & $n=43$ & $n=37$ & \\
\hline Median age at diagnosis of BM (years) & $51(34-74)$ & $56(28-8 \mid)$ & \\
\hline Patients $\geqslant 65$ years & $6(14 \%)$ & $8(21.6 \%)$ & NS \\
\hline Karnofsky Performance Score (median) & 80 & 80 & \\
\hline Patients KPS > $70(\%)$ & $34(79 \%)$ & $28(75.7 \%)$ & NS \\
\hline Hormone receptor positive & $22(51.2 \%)$ & $16(43.2 \%)$ & NS \\
\hline HER2 positive & $43(100 \%)$ & $37(100 \%)$ & NS \\
\hline Grading 3 & $30(69.8 \%)$ & $28(75.7 \%)$ & NS \\
\hline Invasive ductal carcinoma & $35(81.4 \%)$ & $31(83.8 \%)$ & NS \\
\hline \multicolumn{4}{|l|}{ Stage at primary diagnosis } \\
\hline Localised & $35(81.4 \%)$ & $33(89.2 \%)$ & NS \\
\hline Metastatic & $8(\mid 8.6 \%)$ & $4(10.8 \%)$ & NS \\
\hline Visceral disease & $30(69.8 \%)$ & $26(70.3 \%)$ & NS \\
\hline$>2$ Metastatic sites outside the CNS & $23(53.5 \%)$ & $16(43.2 \%)$ & NS \\
\hline Only BM & $3(7 \%)$ & $6(16.2 \%)$ & NS \\
\hline $1-3$ Brain metastases & $23(53.5 \%)$ & $17(45.9 \%)$ & NS \\
\hline Development of BM in $<12$ months & $14(32.6 \%)$ & $9(24.3 \%)$ & NS \\
\hline Radiosurgery or boost without WBRT & $6(14 \%)$ & I $(2.7 \%)$ & NS \\
\hline
\end{tabular}

Abbreviations: $\mathrm{BM}=$ brain metastases; $\mathrm{CNS}=$ central nervous system; $\mathrm{WBRT}=$ whole brain radiotherapy; KPS = Karnofsky Performance Score. ${ }^{a}$ Fisher's exact test. 


\section{Outcome}

Median OS in the total population of 80 patients (groups $\mathrm{A}$ and $\mathrm{B}$ combined) was 10 months (95\% CI: 6.31-13.69).

Subgroup analysis: In patients receiving trastuzumab after completion of local therapy for BM, median OS was 13 months (95\% CI: $8.85-17.15)$. Corresponding numbers were 9 months (95\% CI: $0-20.69)$ in patients treated with chemotherapy, and 3 months (95\% CI: $2.37-3.63)$ in patients with radiotherapy alone, respectively. After a median follow-up of 24 months (range 8-46 months), median OS was not reached in the lapatinib group (Figure 2).

In the univariate model, trastuzumab after completion of local therapy resulted in a significant prolongation of OS compared with

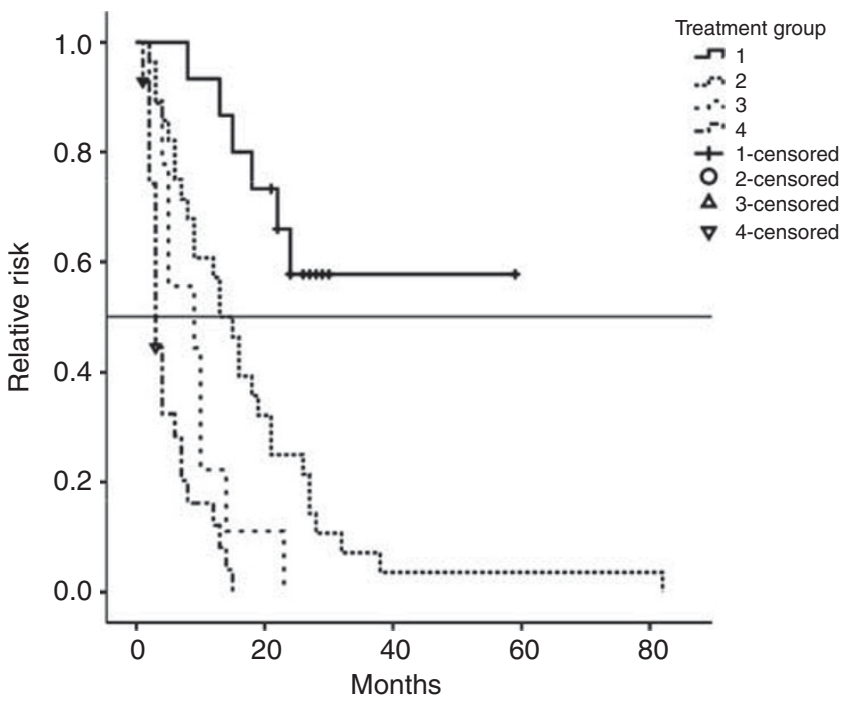

Figure 2 OS (overall survival) after local treatment for brain metastases in all patients (months). Group I: Patients who received lapatinib and trastuzumab with or without chemotherapy after local treatment for brain metastases, $n=15$. Group 2: Patients who received trastuzumab with or without chemotherapy after local treatment for brain metastases, $n=28$. Group 3: Patients who received chemotherapy without anti-HER2 therapy after local treatment for brain metastases, $n=9$. Group 4: Patients without further systemic therapy after local treatment for brain metastases, $n=28$. the control group of 37 patients without anti-HER2 targeted treatment after radiotherapy $(P<0.001)$. Addition of lapatinib again led to a significant prolongation of survival over trastuzumab-based treatment plus/minus chemotherapy $(P=0.002)$.

Other factors associated with OS in the entire population of all 80 patients (groups A and B combined) included: hormone receptor status (median OS hormone receptor-negative 8 months vs 13 months in hormone receptor-positive patients; $P=0.033$ ); presence of visceral metastases (median OS in patients with visceral metastases 9 months $v s 12$ months in those without; $P=0.011$ ); early development of BM (i.e., within 12 months from primary diagnosis of breast cancer or diagnosis of metastatic disease $v s$ later) (median OS in patients with early development of BM 6 months $v s 12$ months; $P=0.057$ ); KPS (median OS KPS $>70$ 13 months vs 4 months in patients with $\mathrm{KPS}=70 ; P=0.001$ ); presence of one to three BM (median OS 18 months vs 4 months in patients with $>3 \mathrm{BM} ; P<0.001$ ).

After correction for those factors, anti-HER2 targeted therapy remained a highly significant predictor for longer OS in the Cox regression model of all 80 patients (HR 0.29; 95\% CI: 0.16-0.54; $P<0.001$ ). Presence of one to three BM (HR 0.32; 95\% CI: $0.18-$ $0.58 ; P<0.001$ ) as well as higher KPS (HR 0.4; 95\% CI: $0.23-0.72$; $P=0.002$ ) remained also significantly associated with better survival in the multivariate comparisons (Table 2).

In patients treated with anti-HER2 targeted therapy after completion of local treatment $(n=43$; group A alone), median OS was 18 months (95\% CI: 12.49-23.51). Factors associated with OS in the univariate analysis included: Presence of visceral metastases (median OS 15 vs 32 months; $P<0.001$ ); more than two metastatic sites (median OS 15 vs 21 months; $P=0.017$ ); KPS (median OS KPS $>7021$ months vs 7 months; $P=0.015$ ); presence of one to three BM (median OS 22 vs 8 months; $P<0.001)$.

After correction for those factors, additional treatment with lapatinib after completion of local therapy remained a significant predictor of longer OS (HR 0.279; 95\% CI: $0.1-0.76 ; P=0.012$ ). Again, presence of one to three $\mathrm{BM}$ was also significantly associated with superior outcomes (HR 0.152; 95\% CI: 0.06-0.41; $P<0.001$; Table 3).

\section{DISCUSSION}

Breast cancer is currently the second most common cause of CNS metastases among all solid cancers. Therefore, BM represent an

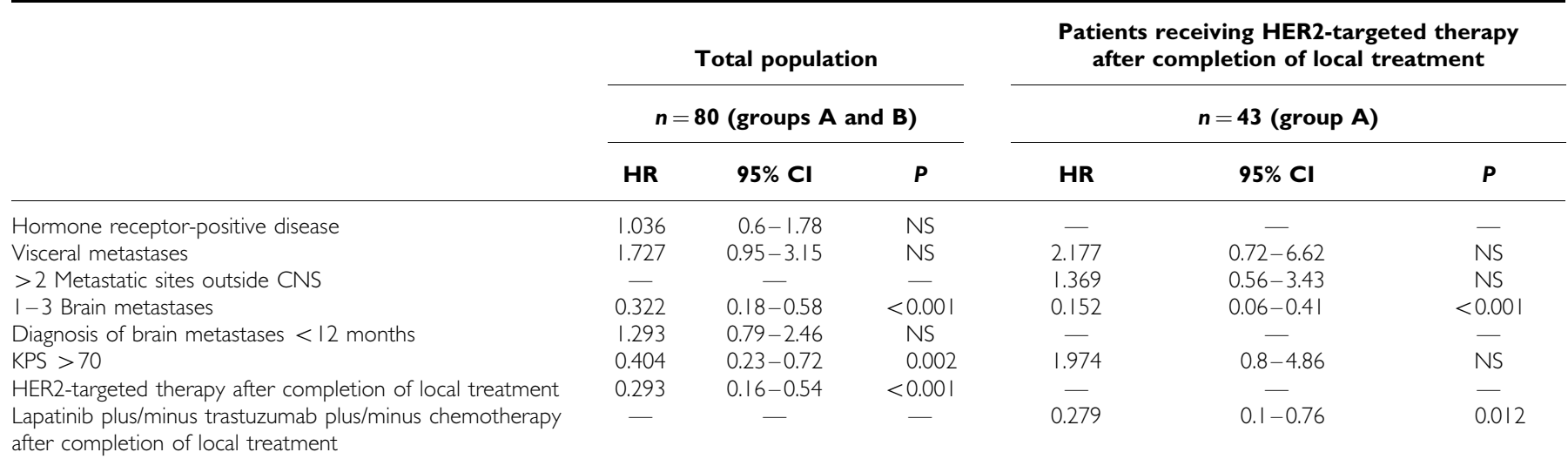

Abbreviations: $\mathrm{HR}=$ hazard ratio; CNS = central nervous system; KPS = Karnofsky Performance Score; $\mathrm{Cl}=$ confidence interval. ${ }^{\text {aHRs, }} \mathrm{Cls}$, and P-values are only provided for variables significantly associated with overall survival in the univariate analysis and were therefore included into the Cox Regression Model. 
important cause of morbidity and mortality in breast cancer patients (Lin and Winer, 2007). An increase in the incidence of BM was observed in recent years, which is usually attributed to the introduction of trastuzumab-based therapy (Bendell et al, 2003; Clayton et al, 2004; Shmueli et al, 2004; Burstein et al, 2005; Viani et al, 2007). Recently, it was reported that up to $40 \%$ of all HER2positive metastatic breast cancer patients will be eventually diagnosed with BM (Bartsch et al, 2009). Other studies, however, suggest that even in the absence of trastuzumab, women with HER2-positive metastatic breast cancer are at increased risk for $\mathrm{BM}$ as compared with women with HER2-negative disease (Pestalozzi and Brignoli, 2000; Pestalozzi et al, 2006). Those divergent results may be explained by the fact that HER2-positive disease has a natural propensity to $\mathrm{BM}$; that effect cannot be fully overcome by trastuzumab, as the antibody, due to its large molecular size, cannot pass through an intact blood-brain barrier and therefore does not offer potential for prophylaxis (Pestalozzi and Brignoli, 2000; Stemmler et al, 2007). Under conditions of an impaired blood-brain barrier such as carcinomatous meningitis or radiotherapy, however, trastuzumab levels within the cerebrospinal fluid are increased, thus serving as rational for continuing trastuzumab after diagnosis of $\mathrm{BM}$ (Stemmler et al, 2007).

BM are usually diagnosed relatively late in the course of metastatic breast cancer; therefore, they commonly occur in patients with advanced systemic disease, as shown in this study. For that reason, it was observed that most patients with BM are in fact dying from systemic disease progression as specific cause of cancer death rather than from BM (Engel et al, 2003). This assumption is strengthened by data from two observations showing that in the absence of trastuzumab after diagnosis of brain metastasis, shorter survival results in HER2-positive as compared with HER2-negative patients, again highlighting the intrinsic aggressiveness of the HER2-positive phenotype and the importance of systemic disease control (Kirsch et al, 2005; Tham et al, 2006).

Since the introduction of trastuzumab, however, various publications reported superior outcomes in terms of OS in patients receiving further systemic therapy, particularly when trastuzumab was included (Bartsch et al, 2007; Dawood et al, 2008; Park et al, 2009). As no significant impact of trastuzumab on the progression of $\mathrm{BM}$ could be identified, we suggest that the main impact of trastuzumab on survival results from improved systemic disease control. Indeed, continuation of trastuzumab exhibits activity even in patients progressing during prior trastuzumab-based therapies (Von Minckwitz et al, 2009). In line with this assumption, lack of systemic disease control was obviously the major limiting factor for survival of women before the trastuzumab era, as the proportion of patients dying from brain lesions instead of systemic disease progression has increased since the introduction of trastuzumab (Kirsch et al, 2005; Lin and Winer, 2007). This points to the urgent need to develop further systemic treatment options exhibiting increased activity in CNS metastases.

Lapatinib, a dual tyrosine-kinase inhibitor of EGFR and HER2 has been approved for the treatment of patients with HER2positive metastatic breast cancer patients progressing during trastuzumab-based therapy (Geyer et al, 2006). Due to the fact that lapatinib is a small molecule, it may penetrate the blood brain barrier and exhibit prophylactic as well as therapeutic effects. Indeed, Lin et al (2008) could demonstrate evidence of modest activity of lapatinib as single agent in 242 patients pretreated with trastuzumab and with progressive CNS metastases after radiotherapy ( $R R$ in brain 6\%). In the lapatinib plus capecitabine extension phase of that study, a $20 \%$ response rate within the CNS was observed (Lin et al, 2009). In line with those data, a study recently published even suggested a beneficial impact of lapatinib-based therapy on OS: Significantly longer OS was observed in 30 patients receiving lapatinib and capecitabine after diagnosis of $\mathrm{BM}$ as compared with a similar population receiving trastuzumab-based therapies alone (27.9 months $v s 16.7$ months; $P=0.01$ ) (Metro et al, 2011). In that trial, however, not all patients received local treatment for CNS lesions; furthermore, no correction for potential confounders of survival such as KPS or number of BM was performed.

In general, our data fit well into the spectrum of previous studies: patients receiving ongoing systemic therapy showed a marked increase in survival over patients without any further systemic treatment. Patients receiving trastuzumab after completion of local therapy had a median OS of 13 months, which was significantly longer than with chemotherapy alone. In patients receiving lapatinib-based therapy, median OS was still not reached after 24 months of follow-up. This resulted in a significant prolongation of survival over trastuzumab-based therapy alone $(P=0.002)$. To correct for other factors significantly associated with OS in the univariate analysis, a Cox proportional hazard models was applied. In that analysis, additional treatment with lapatinib retained significance as predictor of superior outcome (HR for death $0.279 ; 95 \% \mathrm{CI}: 0.1-0.76 ; P=0.012$ ). Presence of one to three $\mathrm{BM}$ was also significantly associated with improved outcome (HR 0.152; 95\% CI: $0.06-0.41 ; P<0.001$ ). In line with our observation, Lee et al (2008) reported that after completion of local therapy for CNS metastases, ongoing chemotherapy, performance status as well as the number of BM were independent predictors of survival. Similar results were reported by Lentzsch et al (1999). In difference to those studies, however, no significant impact of performance status was observed in our analysis. Two facts may add to this observation: first, this was a relatively homogenous population of patients with good performance status, as only 18 out of 80 patients had KPS $<80(22.5 \%)$. Furthermore, we suggest that active systemic treatment even in the presence of BM may improve outcome to a point, which reduces the impact of baseline performance status on survival. Indeed, in the overall population of 80 patients, high KPS retained statistical significance as predictor of survival (KPS >70: HR 0.4; 95\% CI: $0.23-0.72$; $P=0.002$ ).

Our study is clearly limited by its retrospective nature and single institutional design. However, it represents a relatively homogeneous group of patients presenting with KPS $\geqslant 70$, all of whom received optimal local therapy for $\mathrm{BM}$. The imbalance in the percentage of patients treated with radiosurgery or boost irradiation alone is not expected to impact upon survival (Aoyama et al, 2006).

In conclusion, our results indicate a significant improvement of OS in patients with BM from HER2-positive breast cancer when treated in addition with an anti-HER2 agent, especially lapatinib. Impact of lapatinib retained statistical significance after correction for other potential confounders of OS, such as presence of visceral metastases, number of extracranial metastatic sites, KPS and number of BM.

\section{Conflict of interest}

$\mathrm{RB}$ has received lecture honoraria from Glaxo-Smith-Kline and Hoffmann-La Roche. MG has received lecture honoraria from Hoffmann-La Roche; has served on advisory board of HoffmannLa Roche. CCZ has received lecture honoraria from Glaxo-SmithKline and Hoffmann-La Roche; has served on advisory boards of Glaxo-Smith-Kline and Hoffmann-La Roche. GGS has received lecture honoraria from Glaxo-Smith-Kline and Hoffmann-La Roche; has served on advisory boards of Glaxo-Smith-Kline and Hoffmann-La Roche. The remaining authors declare no conflict of interest. 


\section{REFERENCES}

Aoyama H, Shirato H, Tago M, Nakagawa K, Toyoda T, Hatano K, Kenjyo M, Oya N, Hirota S, Shioura $H$, Kunieda E, Inomta $T$, Hayakawa K, Katoh N, Kobashi G (2006) Stereotactic radiosurgery plus whole-brain radiation therapy $v s$ stereotactic radiosurgery alone for treatment of brain metastases: a randomized controlled trial. JAMA 295: $2483-2491$

Bartsch R, De Vries C, Pluschnig U, Dubsky P, Bago-Horvath Z, Gampenrieder SP, Rudas M, Mader RM, Rottenfusser A, Wiltschke C, Gnant M, Zielinski CC, Steger GG (2009) Predicting for activity of second-line trastuzumab-based therapy in her2-positive advanced breast cancer. BMC Cancer 9: 367

Bartsch R, Rottenfusser A, Wenzel C, Wenzel C, Dieckmann K, Pluschnig U, Altorjai G, Rudas M, Mader RM, Poetter R, Zielinski CC, Steger GG (2007) Trastuzumab prolongs overall survival in patients with brain metastases from HER2 positive breast cancer. J Neurooncol 85: $311-317$

Bendell JC, Domchek SM, Burstein HJ, Harris L, Younger J, Kuter I, Bunnell C, Rue M, Gelman R, Winer E (2003) Central nervous system metastases in women who receive trastuzumab-based therapy for metastatic breast carcinoma. Cancer 97: 2972-2977

Bindal RK, Samaya R, Leavens ME, Lee JJ (1993) Surgical treatment of multiple brain metastases. J Neurosurg 79: 210-216

Borgelt B, Gelber R, Kramer S, Brady LW, Chang CH, Davis LW, Perez CA, Hendrickson FR (1980) The palliation of brain metastases: final results of the first two studies by the Radiation Therapy Oncology Group. Int $J$ Radiat Oncol Biol Phys 6: 1-9

Broadbent AM, Hruby G, Tin MM, Jackson M, Firth I (2004) Survival following whole brain radiation treatment for cerebral metastases: an audit of 747 patients. Radiother Oncol 71: 259-265

Burstein HJ, Lieberman G, Slamon DJ, Winer EP, Klein P (2005) Isolated central nervous system metastases in patients with Her2-overexpressing breast cancer treated with first-line trastuzumab-based therapy. Ann Oncol 16: $1772-1777$

Cameron D, Casey M, Press M, Lindquist D, Pienkowski T, Romieu CG, Chan S, Jagiello-Gruszfeld A, Kaufman B, Crown J, Chan A, Campone M, Viens P, Davidson N, Gorbounova V, Raats JI, Skarlos D, Newstat B, Roychowdhury D, Paoletti P, Oliva C, Rubin S, Stein S, Geyer CE (2008) A phase III randomized comparison of lapatinib plus capecitabine $v s$ capecitabine alone in women with advanced breast cancer that has progressed on trastuzumab: updated efficacy and biomarker analyses. Breast Cancer Res Treat 112: 533 - 543

Clayton AJ, Danson S, Jolly S, Ryder WD, Burt PA, Stewart AL, Wilkinson PM, Welch RS, Magee B, Wilson G, Howell A, Wardley AM (2004) Incidence of cerebral metastases in patients treated with trastuzumab for metastatic breast cancer. Br J Cancer 91: 639-643

Dawood S, Broglio K, Esteva FJ, Ibrahim NK, Kau SW, Islam R, Aldape KD, Yu TK, Hortobagyi GN, Gonzalez-Angulo AM (2008) Defining prognosis for women with breast cancer and CNS metastases by HER2 status. Ann Oncol 19: $1242-1248$

Engel J, EcKel R, Aydemir U, Aydemir S, Kerr J, Schlesinger-Raab A, Dirschedl P, Hoelzel D (2003) Determinants and prognoses of locoregional and distant progression in breast cancer. Int J Radiat Oncol Biol Phys 55: $1186-1195$

Evans A, James J, Cornford E, Chan SY, Burrell HC, Pinder SE, Gutteridge E, Robertson JF, Hornbuckle J, Cheung KL (2004) Brain metastases from breast cancer: identification of a high risk group. Clin Oncol 16: 345-349

Geyer CE, Forster J, Lindquist D, Chan S, Romieu CG, Pienkowski T, Jagiello-Gruszfeld A, Crown J, Chan A, Kaufman B, Skarlos D, Campone M, Davidson N, Berger M, Oliva C, Rubin SD, Stein S, Cameron D (2006) Lapatinib plus capecitabine for HER2-positive advanced breast cancer. $N$ Engl J Med 355: 2733-2743

Kirsch DG, Ledezma CJ, Mathews CS, Bhan AK, Ancukiewicz M, Hochberg FH, Loeffler JS (2005) Survival after brain metastases from breast cancer in the trastuzumab era. J Clin Oncol 23: 2114-2116

Lee SS, Ahn JH, Kim MK, Sym SJ, Gong G, Ahn SD, Kim SB, Kim WK (2008) Brain metastases in breast cancer: prognostic factors and management. Breast Cancer Res Treat 111: 523-530

Lentzsch S, Reichardt P, Weber F, Budach V, Dörken B (1999) Brain metastases in breast cancer: prognostic factors and management. Eur J Cancer 35: $580-585$

Lin N, Bellon J, Winer E (2004) CNS metastases in breast cancer. J Clin Oncol 22: $3608-3617$
Lin NU, Carey LA, Liu MC, Younger J, Come SE, Ewend M, Harris GJ, Bullitt E, Van den Abbeele AD, Henson JW, Li X, Gelman R, Burstein HJ, Kasparian E, Kirsch DG, Crawford A, Hochberg F, Winer EP (2008) Phase II trial of lapatinib for brain metastases in patients with human epidermal growth factor receptor 2-positive breast cancer. J Clin Oncol 26: 1993 - 1999

Lin NU, Dieras V, Paul D, Lossignol D, Christodoulou C, Stemmler HJ, Roché H, Liu MC, Greil R, Ciruelos E, Loibl S, Gori S, Wardley A, Yardley D, Brufsky A, Blum JL, Rubin SD, Dharan B, Steplewski K, Zembryki D, Oliva C, Roychowdhury D, Paoletti P, Winer EP (2009) Multicenter phase II study of lapatinib in patients with brain metastases from HER2-positive breast cancer. Clin Cancer Res 15: $1452-1459$

Lin NU, Winer EP (2007) Brain metastases: the HER2 paradigm. Clin Cancer Res 13: $1648-1655$

Lohr F, Pirzkall A, Hof H, Fleckenstein K, Debus J (2001) Adjuvant treatment of brain metastases. Semin Surg Oncol 20: 50-56

Lower EE, Dosick DR, Blau R, Brennan L, Danneman W, Hawley DK (2003) Increased rate of brain metastasis with trastuzumab therapy not associated with impaired survival. Clin Breast Cancer 4: $114-119$

Lutterbach J, Bartelt S, Stancu E, Guttenberger R (2002) Patients with brain metastases: hope for recursive partitioning analysis (RPA) class 3 . Radiother Oncol 263: 399-405

Metro G, Foglietta J, Russillo M, Stocchi L, Vidiri A, Giannarelli D, Crino L, Papaldo P, Mottolese M, Cognetti F, Fabi A, Gori S (2011) Clinical outcome of patients with brain metastases from HER2-positive breast cancer treated with lapatinib and capecitabine. Ann Oncol 22: $625-630$

Miller KD, Weathers T, Haney LG, Timmerman R, Dickler M, Shen J, Sledge Jr GW (2003) Occult central nervous system involvement in patients with metastatic breast cancer prevalence, predictive factors and impact on overall survival. Ann Oncol 14: $1072-1077$

Park YH, Park MJ, Ji SH, Yi SY, Lim DH, Nam DH, Lee J-I, Park W, Choi DH, Huh SJ, Ahn JS, Kang WK, Park K, Im Y-H (2009) Trastuzumab treatment improves brain metastasis outcomes through control and durable prolongation of systemic extracranial disease in HER2-overexpressing breast cancer patients. Br J Cancer 100: $894-900$

Pestalozzi BC, Brignoli S (2000) Trastuzumab in CSF. J Clin Oncol 18: 2349- 2351

Pestalozzi BC, Zahrieh D, Price KN, Holmberg SB, Lindtner J, Collins J, Crivellari D, Fey MF, Murray E, Pagani O, Simoncini E, Castiglione-Gertsch M, Gelber RD, Coates AS, Goldhirsch A, International Breast Cancer Study Group (IBCSG) (2006) Identifying breast cancer patients at risk for central nervous system (CNS) metastases in trials of the international breast cancer study group (IBCSG). Ann Oncol 17: $935-944$

Rosner D, Nemoto T, Lane WW (1986) Chemotherapy induces regression of brain metastases in breast carcinoma. Cancer 58: $832-839$

Shmueli E, Wigler N, Inbar M (2004) Central nervous system progression among patients with metastatic breast cancer responding to trastuzumab treatment. Eur J Cancer 40: 379-382

Slimane K, Andre F, Delaloge S, Dunant A, Perez A, Grenier J, Massard C, Spielmann M (2004) Risk factors for brain relapse in patients with metastatic breast cancer. Ann Oncol 15: $1640-1644$

Stemmler HJ, Heinemann V (2008) Central nervous system metastases in Her2-overexpressing metastatic breast cancer: a treatment challenge. Oncologist 13: 739-750

Stemmler HJ, Schmitt M, Willems A, Bernhard H, Harbeck N, Heinemann V (2007) Ratio of trastuzumab levels in serum and cerebrospinal fluid is altered in HER2-positive breast cancer patients with brain metastases and impaired blood-brain barrier. Anticancer Drugs 18: $23-28$

Tham YL, Sexton K, Kramer R, Hilsenbeck S, Elledge R (2006) Primary breast cancer phenotypes associated with propensity for central nervous system metastases. Cancer 107: 696-704

Viani GA, Afonso SL, Stefano EJ, De Fendi LI, Soares FV (2007) Adjuvant trastuzumab in the treatment if her-2-positive early breast cancer: a meta-analysis of published randomized trials. BMC Cancer 7: 153 
Von Minckwitz G, du Bois A, Schmidt M, Maass N, Cufer T, de Jongh FE, Maartense E, Zielinski C, Kaufmann M, Bauer W, Baumann KH, Clemens MR, Duerr R, Uleer C, Andersson M, Stein RC, Nekljudova V, Loibl S (2009) Trastuzumab beyond progression in human epidermal growth factor receptor 2-positive advanced breast cancer: a German breast group 26/breast international group 03-05 study. J Clin Oncol 27: 1999-2006
Weil RJ, Palmieri DC, Bronder JL, Stark AM, Steeg PS (2005) Breast cancer metastasis to the central nervous system. Am J Pathol 167: 913-920

Yau T, Swanton C, Chua S, Sue A, Walsh G, Rostom A, Johnston SR, O'Brien ME, Smith IE (2006) Incidence, pattern and timing of brain metastases among patients with advanced breast cancer treated with trastuzumab. Acta Oncol 45: 196-201

This work is published under the standard license to publish agreement. After 12 months the work will become freely available and the license terms will switch to a Creative Commons Attribution-NonCommercial-Share Alike 3.0 Unported License. 